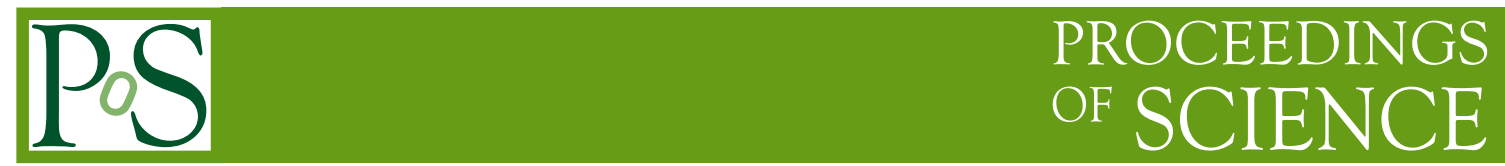

\title{
Quantum coherent measurements
}

\author{
Roy A. Briere* ${ }^{\dagger}$ \\ Carnegie Mellon University \\ E-mail: rrorieredandrew.cmu,edu
}

The basic physics of quantum-correlated $D^{0}, \bar{D}^{0}$ pairs produced at threshold via decays of the $\psi(3770)$ is introduced. The connection to extractions of the CKM angle $\gamma\left(\phi_{3}\right)$ from $B$ decays is emphasized throughout. Recent quantum correlation results from BESIII and CLEO-c are then summarized before closing with a discussion of selected issues.

VIII International Workshop On Charm Physics

5-9 September, 2016

Bologna, Italy

* Speaker.

${ }^{\dagger}$ Work supported by the US DOE under Contract No. DESC0010118. 


\section{Introduction}

An $e^{+} e^{-}$collider running at the $\psi(3770)$ resonance near open-charm $(D \bar{D})$ threshold will produce entangled neutral $D$ meson pairs. This entanglement, or quantum coherence, leads to a variety of interesting effects and in particular allows convenient access to certain relative phases. This phenomenon has been known for some time [四] and by now consequences have been explored in detail [0, [1, 田, ] ]. It is quite interesting to observe these EPR-like quantum correlation effects in an HEP experiment.

One major goal of quark flavor physics involves over-constraining the CKM mixing matrix in an effort to find evidence for new physics. Measurements of the CKM angle $\gamma$ (or $\phi_{3}$ ) may in principle be made with negligible theoretical uncertainty using $B \rightarrow D K$ decays [ [G, 四]. Indeed, several experiments are already exploiting this via analyses of a wide variety of related decay chains [8]].

In order to perform clean extractions of $\gamma$, one must avoid introducing unnecessary modeldependence in the analyses. Data from charm threshold can be used to to avoid such pitfalls, as we will see below, by directly measuring the strong-phase quantities that the $B$ decay analyses need. Such external inputs from charm are useful in avoiding ambiguities, simplifying analyses and reducing uncertainties. They also have the virtue of replacing hard-to-evaluate uncertainties from model dependence with clearer and largely statistical uncertainties from the threshold charm results.

Quantum-correlated effects appear in three places: (i) correlated charm at threshold, (ii) $B \rightarrow$ $D K$ analyses with common $D$ final states, and (iii) charm mixing. Most of this review concerns using (i) as an input to (ii). At the end, we will briefly cite discussions of using (i) as an input to (iii), and (iii) and an input to (ii).

\section{Charm at Threshold and Quantum Coherence}

Production of the $\psi(3770)$ state is followed about half of the time by decay into a neutral $D$ meson pair which is entangled as:

$$
\psi(3770) \rightarrow \frac{1}{\sqrt{2}}\left[D^{0}(+z) \bar{D}^{0}(-z)-\bar{D}^{0}(+z) D^{0}(-z)\right]
$$

or, using the $C P$ eigenstate combinations $D_{C P \pm}=\left[D^{0} \pm \bar{D}^{0}\right] / \sqrt{2}$

$$
\psi(3770) \rightarrow \frac{1}{\sqrt{2}}\left[D_{C P-}(+z) D_{C P+}(-z)-D_{C P+}(+z) D_{C P-}(-z)\right]
$$

In both cases, the (arbitrary) center-of-mass decay axis is labelled as $\pm z$. Experiments measure various combinations of rates, such as the total rate for one $D$ decay final state (inclusive "single tags") or for specific pairs of $D$ decay modes (exclusive "double tags"). These rates display some very interesting effects arising from quantum coherence and the resulting interference. In particular, the double-tag rates have useful sensitivities while single-tag rates provide useful for normalizations.

One convenient way to introduce interference effects involves observing one $D$ meson decaying to a $C P$ eigenstate; this projects the other meson into a coherent $D^{0}, \bar{D}^{0}$ superposition with the 
opposite $C P$, as displayed in Eqn. 22 . If that second $D$ decays to a mode accessible to both the $D^{0}$ and $\bar{D}^{0}$ components of the state, then there will be interference. One can even change the sign of the interference term by choosing $C P-$ instead of $C P+$ eigenstates for the first decay. While this is a nice example, it is important to realize that the participation of $C P$ eigenstates is not essential. There is coherence in the $D$-pair wavefunction no matter which basis is chosen and this leads in general to interference effects.

It is useful to classify some key types of $D$ meson decays. Cabibbo-favored (CF) decays result from $c \rightarrow s W^{+}, W^{+} \rightarrow u \bar{d}$ transitions, producing one ${ }^{1} \bar{K}$ meson $\left(\right.$ a $K^{-}$or $\bar{K}^{0}$ ). Singly-Cabibbosuppressed (SCS) decays result from $c \rightarrow s W^{+}, W^{+} \rightarrow u \bar{s}$ and $c \rightarrow d W^{+}, W^{+} \rightarrow u \bar{d}$ transitions, resulting in an even number (0 or 2 ) of kaons. Doubly-Cabibbo-suppressed decays (DCSD) are from the process $c \rightarrow d W^{+}, W^{+} \rightarrow u \bar{s}$ resulting in a "wrong-sign" $K$ meson. Actually, "wrongstrangeness" is a more appropriate terminology; however when the single kaon produced is electrically charged, then CF (DCSD) decays of $D$ mesons produce a $K^{-}\left(K^{+}\right)$, and the opposite for $\bar{D}$ decays. There is a suppression of other decays relative to CF ones controlled by the Cabibbo angle, $\theta_{C}$ : SCS decays are suppressed in amplitude by one power of $\tan \theta_{C} \sim 0.22$, while DCSD suffer from two powers of this same factor.

In addition to the Cabibbo factors corresponding to a given $D$ decay, certain other properties are also worth noting. Hadronic decays refer to the cases where all decay products are mesons, while semileptonic means that there is a charged lepton and neutrino $\left(e v_{e}, \mu v_{\mu}\right)$ produced along with one or more hadrons. Some final states are symmetric (self-conjugate) with respect to particleantiparticle exchange, while others are not. Also, some final states are "flavored", meaning that one can infer whether the initial state contained a $c$ or $\bar{c}$ quark. Such flavor-tagging is valid only at the time of decay, due to $D^{0}-\bar{D}^{0}$ mixing. Furthermore, it is only exact for semileptonic decays: for CF hadronic decays, flavor tagging is contaminated by the rarer DCSD processes.

The various $D$ decays relevant to our discussions are summarized in Table $\square$. We note that it possible to be neither self-conjugate nor flavored but it is not possible to be both.

\begin{tabular}{|l|l|l|}
\hline Type & Examples & Amplitude(s) \\
\hline \hline Flavored & & \\
\hline - Flavored semileptonic & $K^{-} e^{+} v, K^{-} \mu^{+} v$ & Pure CF \\
- Flavored hadronic & $K^{-} \pi^{+}, K^{-} \pi^{+} \pi^{0}, K^{-} \pi^{+} \pi^{+} \pi^{-}$ & CF + DCSD \\
\hline \hline Self-conjugate & & \\
\hline - 2-body CP eigenstate & $K^{-} K^{+}, \pi^{+} \pi^{-}, K_{S} \pi^{0}$ & SCS \\
- Multi-body I & $K_{S} h^{+} h^{-}, K_{L} h^{+} h-$ & CF + DCSD \\
- Multi-body II & $K^{+} K^{-} \pi^{+} \pi^{-}, \pi^{+} \pi^{-} \pi^{0}$ & SCS \\
\hline \hline Neither & $K_{S} K^{-} \pi^{+}, K_{S} K^{-} \pi^{+} \pi^{0}$ & SCS \\
\hline \hline Both & - not logically possible- & \\
\hline
\end{tabular}

Table 1: A summary of relevant classes of $D$ decays; $h$ indicates $\pi$ or $K$. "Neither" and "both" refer to being or not being flavored and self-conjugate.

\footnotetext{
${ }^{1}$ Or, rarely, producing $\bar{K} K \bar{K}$, but such decays are not relevant for us here.
} 


\section{Multi-Body Coherence Factors}

Consider the interference of two two-body amplitudes:

$$
\left|\mathscr{A}_{1}+\mathscr{A}_{2}\right|^{2}=\left|\mathscr{A}_{1}\right|^{2}+\left|\mathscr{A}_{2}\right|^{2}+2\left|\mathscr{A}_{1}\right|\left|\mathscr{A}_{2}\right| \cos \delta=A_{1}^{2}+A_{2}^{2}+2 A_{1} A_{2} \cos \delta
$$

where 1 and 2 label, for example, the CF and DCSD $D^{0} \rightarrow K^{\mp} \pi^{ \pm}$amplitudes, and $\delta$ is their relative phase. The second equality uses the magnitudes of the amplitudes, $A_{i}=\left|\mathscr{A}_{i}\right|$. The generalization of this to multi-body decays results in the introduction of Atwood-Soni coherence factors [ $[$ ] . In this case, one has to integrate over the Dalitz plot. After doing so, these coherence factors allow one to construct an expression very similar to the two-body relation.

We will need to distinguish between the complex amplitudes at one point in phase space (denoted by $x), \mathscr{A}_{i}(x)$, and their real phase-space-averaged integrals, $A_{i}$, defined via $A_{i}^{2}=\int d x\left|\mathscr{A}_{i}\right|^{2}$. These generalize the simple $A_{i}=\left|\mathscr{A}_{i}\right|$ relation of the two-body case. For multi-body cases, we have:

$$
\int d x\left|\mathscr{A}_{1}+\mathscr{A}_{2}\right|^{2}=A_{1}^{2}+A_{2}^{2}+2 R A_{1} A_{2} \cos \delta
$$

Here, the coherence factor is given by $R$ and $\delta$ which summarize the net effect of amplitude and phase variation across the Dalitz plot. Using $D^{0} \rightarrow K^{\mp}(n \pi)^{ \pm}$decays as an example:

$$
R_{K n \pi} e^{-i \delta_{K n \pi}}=\frac{\int d x \mathscr{A}_{K^{-}(n \pi)^{+}} \mathscr{A}_{K^{+}(n \pi)^{-}}}{A_{K^{-}(n \pi)^{+}} A_{K^{+}}(n \pi)^{-}}
$$

where we have simply taken the ratio of the correct cross-term to the simplified average form used in Eqn. 3.2. In a two-body decay $R=1$ and there is only a relative phase $\delta$ which controls the Dalitz-averaged CF-DCSD interference. In the multi-body case, there are two real parameters remaining: $R, \delta$. But we can also use these parameters to discuss decays beyond $K^{-}(n \pi)^{+}$.

In general, we expect the parameter $R<1$ due to two effects. First, the $x$-dependent phase relative phase $\arg \left(\mathscr{A}_{2}^{*}(x) \mathscr{A}_{1}(x)\right)$ varies as we integrate. Second, the amplitudes don't track each other in magnitude across phase space; i.e., the local amplitude ratio $\left|\mathscr{A}_{2}(x) / \mathscr{A}_{1}(x)\right|$ is not constant.

Viewed as polar coordinates, $R, \delta$ lie on or inside the unit circle. It is also possible to consider a Cartesian basis, $(c, s)=(R \cos \delta, R \sin \delta)$. One can also obtain results in separate sub-regions, or bins, of the Dalitz plot, labeled by a subscript $i$. As we will see below, one typically uses (for various reasons) $R, \delta$ for $K^{-}(n \pi)^{+}$and several bins of $c_{i}, s_{i}$ for $K_{S} \pi^{+} \pi^{-}$and related modes.

\section{Quantum Correlations for Pedestrians}

The simplest quantum correlation effect in $\psi(3770)$ decays involves both mesons decaying to $C P$-eigenstates. Like- $C P(++,--)$ combinations are forbidden, while opposite- $C P(+-,-+)$ are enhanced by two, as one can see directly from the wave function in Eqn. 2.2. However, as noted above, interference effects are quite general. We denote the decay amplitudes as $\mathscr{A}\left(D^{0} \rightarrow F\right)=\mathscr{A}_{F}$ and $\mathscr{A}\left(\bar{D}^{0} \rightarrow F\right)=\overline{\mathscr{A}}_{F}$. For the real, averaged amplitudes defined above, we have $\bar{A}_{F}=A_{\bar{F}}$ and $\bar{A}_{\bar{F}}=A_{F}$. Then, the decay width for a double-tag final state to modes $F, G$ is given by:

$$
\Gamma_{F G}=\Gamma_{0}\left[A_{F}^{2} \bar{A}_{G}^{2}+\bar{A}_{F}^{2} A_{G}^{2}-2 A_{F} \bar{A}_{F} A_{G} \bar{A}_{G} R_{F} R_{G} \cos \left(\delta_{G}-\delta_{F}\right)\right]
$$


If, for example, we take $F=K^{-} \pi^{+} \pi^{0}, G=K^{-} \pi^{+} \pi^{+} \pi^{-}$, then factoring out the larger amplitudes gives

$$
\Gamma_{F G}=\Gamma_{0} A_{F}^{2} A_{G}^{2}\left[r_{F}^{2}+r_{G}^{2}-2 r_{F} r_{G} R_{F} R_{G} \cos \left(\delta_{G}-\delta_{F}\right)\right]
$$

Here, $r_{F, G}=\bar{A}_{F, G} / A_{F, G}$, defined such that $r \leq 1$. For other cases, like opposite-sign $K^{-}(n \pi)^{+}$vs. $K^{+}(n \pi)^{-}$, one would replace $r_{F}^{2}+r_{G}^{2}$ by $1+r_{F}^{2} r_{G}^{2}$ to keep both $r \leq 1$. This form is considerably simplified in many other cases. For example, $r= \pm 1$ for $C P$ eigenstates, $r=0$ for semileptonic decays. But $r \simeq \tan ^{2} \theta_{C} \simeq 0.05$ for CF+DCSD cases. For $K^{\mp}(n \pi)^{ \pm}, R, \delta$ are the Atwood-Soni coherence factors which are a priori unknown, except that $R=1$ for $n=1\left(K^{\mp} \pi^{ \pm}\right)$. They are trivial for $C P$-eigenstates $(R=1, \delta=0, \pi)$ and for semileptonic decays $(R=0)$.

Note that interference in general is only sensitive to $\operatorname{Re}\left(e^{-i \delta}\right)=\cos \delta$. But $\delta$ here is a difference between the two relative phases for the two final states of the double-tag. If one decay mode has a trivial phase, then is it true that one will only be sensitive to $\cos \delta$ for the other non-trivial amplitude. But if both decays have non-trivial phases, one gets

$$
\cos \left(\delta_{G}-\delta_{F}\right)=\cos \delta_{G} \cos \delta_{F}+\sin \delta_{G} \sin \delta_{F}
$$

One can measure enough observables to separately determine both the sin and cos terms and thereby recover sensitivity to $\sin \delta$ (and hence the sign of $\delta$ ). This can be achieved in the obvious way by employing two different decay modes, for example different $n$ with $K(n \pi)$ modes. But it can also be done with different portions of phase-space in a multi-body decay which act as independent modes. Note that when using $c_{i}, s_{i}$, we must recall that these arise as Cartesian coordinates of some binned $R e^{-i \delta}$, and not $e^{-i \delta}$. Hence, $s_{i}^{2} \neq 1-c_{i}^{2}$ : there are still two independent degrees of freedom.

\section{Charm Threshold and $B$ Physics}

There are a number of methods to extract the CKM angle $\gamma$ from $B \rightarrow D K$ decays, an "alphabet soup" of acronyms, distinguished largely by the specific $D$ decay involved. The key is to exploit a final state which is common to both $D^{0}$ and $\bar{D}^{0}$ decays. We first give a brief summary of methods, and then discuss what charm data at threshold can do to help such analyses.

The pioneering GLW method [प, प] uses $D$ decays to a $C P$ eigenstate. The ADS method [ㅁ] [13], [4]] employs modes that are CF and DCSD; this helps to balance the overall amplitudes to

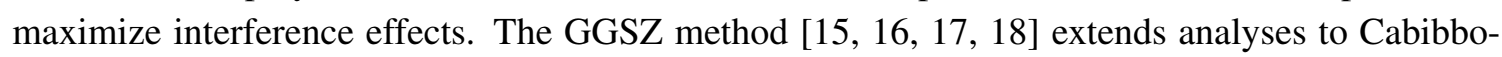
favored self-conjugate multi-body modes. We also note the infrequently mentioned GLS paper [एव], which proposed using SCS decays to non- $C P$-eigenstates (such as $K^{*} K$, which decays to $\left.K_{S} K \pi\right)$. The utility of $B \rightarrow D^{*} K$ in addition to $B \rightarrow D K$ has also been explored [20]. As one sees, $B$ factories (BaBar, Belle, LHCb, and soon BelleII) have many choices of $D$ decay modes to use when studying $\gamma[$ [ $]$ ].

We now quickly survey the charm physics within each $\gamma$ method. Semileptonic $D$ decays are impractical for $B$ physics, but they are useful for charm threshold work. Since there is no interference, they are useful for normalization. For the $C P$-eigenstates of GLW, the strong phase differences between the $D^{0}$ and $\bar{D}^{0}$ amplitudes are always trivial: 0 or $\pi$. There is no need for input from charm threshold studies, but these final states are very useful as one half of double-tag combinations in our threshold charm analyses. 
For the ADS method, there is a non-trivial strong phase between the CF and DCSD amplitudes. Threshold data can provide the necessary Dalitz-averaged Atwood-Soni coherence factors (two parameters in general, or in the two-body $K \pi$ case just one relative phase). These modes are also sometimes used as normalization since they are easier to reconstruct than semileptonic decays, but one must be careful to correctly account for the DCSD effects.

Multi-body self-conjugate modes are the basis of the GGSZ method, and threshold can provide strong-phase information similar to the previous case. In practice, data is presented as Cartesian $c_{i}, s_{i}$ parameters, with $i$ labeling binning of the Dalitz plot. It is important to note that while there is a concept of optimal binning, for best statistical precision, there is no bias caused by any particular choice of binning.

In cases where a multi-body state is dominated by sub-components that are dominantly of one $C P$ value, it is interesting to measure the $C P$-purity of the state: that is the fraction of the dominant $C P$, denoted $F_{+}$for the $C P+$ fraction, etc.

If we examine the role of inputs measured at charm threshold in more detail, there are two general motivations. These are avoiding model dependence and accessing strong phases.

Large and cleanly-separated samples of $D^{0}$ and $\bar{D}^{0}$ decays are available from $B$ factories via $D^{*+} \rightarrow D^{0} \pi^{+}$tagging. Much of the strong phase variation across the Dalitz plot may be studied via commonly-used fits to isobar models or related extensions. However, using such models introduces systematic uncertainties that can be avoided by measuring the quantities of interest directly at charm threshold.

In addition, in some cases, there is a strong phase that is simply not accessible via flavor-tagged $D^{0}, \bar{D}^{0}$ samples alone. Imagine that we fit both CF $D^{0} \rightarrow K^{-} \pi^{+} \pi^{0}$ and DCSD $D^{0} \rightarrow K^{+} \pi^{-} \pi^{0}$ with $N$ isobar amplitudes. Each fit is sensitive to $N-1$ relative phases, so $2 N-2$ relative phases are measured. But at threshold, we can interfere these two process, and since all $2 N$ amplitudes are involved, one is sensitive to $2 N-1$ phases: one more. Said another way, in separate isobar fits, one amplitude in each fit is chosen as real. But there is no linkage between the two fits and thus it is impossible to measure the relative phase of these reference amplitudes.

For the ADS and GLS modes, both issues are relevant. For GGSZ modes, only the modeldependence is relevant: the relative $D^{0}, \bar{D}^{0}$ phase is again trivial as it is for two-body $C P$-eigenstates.

We can summarize the experimental outputs in Table [1.

\begin{tabular}{|c|c|c|c|}
\hline Mode & Method & Observables & References \\
\hline$\overline{K^{-} K^{+}, \pi^{+} \pi^{-}}$ & GLW & - & - \\
\hline$K^{-} \pi^{+}$ & ADS & $\delta$ & {$[21,122,[33]$} \\
\hline$K^{-} \pi^{+} \pi^{0}, K^{-} \pi^{+} \pi^{+} \pi^{-}$ & ADS+ & $R, \delta$ & {$[23,[29,132]$} \\
\hline$K_{S} K^{-} \pi^{+}$ & GLS & $R, \delta$ & [24] \\
\hline$K_{S} \pi^{+} \pi^{-}, K_{S} K^{+} K^{-}$ & GGSZ & $c_{i}, s_{i}$ & $[25], 26,134]$ \\
\hline$\pi^{+} \pi^{-} \pi^{0}, K^{+} K^{-} \pi^{0}, \pi^{+} \pi^{-} \pi^{+} \pi^{-}$ & GLW, GGSZ & $F_{+}$ & $[\mathrm{BO}, \mathrm{B}]]$ \\
\hline$K^{+} K^{-} \pi^{+} \pi^{-}$ & GGSZ & $D^{0}, \bar{D}^{0}$ isobar fits & [28] \\
\hline
\end{tabular}

Table 2: Summary of quantities currently accessed with charm threshold data, arranged by mode and $\gamma$ method, with references. 


\section{A Survey of Results from Charm Threshold}

Results are available from both CLEO-c and BESIII. CLEO-c analyses mainly use $0.818 \mathrm{fb}^{-1}$ of $\psi(3770)$ data $^{2}$, while BESIII uses $2.92 \mathrm{fb}^{-1}$.

We begin by reviewing the CLEO-c results. The $K^{-} \pi^{+}$phase was first reported in 2008 [ㅁ] ] and then updated in 2012 [22]; both versions make use of a complex global analysis and fit. Using external mixing constraints, they find $\cos \delta_{K \pi}=1.115_{-0.17}^{+0.19}{ }_{-0.08}^{+0.00}$. In 2009, Atwood-Soni coherence factors were published for $K^{-} \pi^{+} \pi^{0}$ and $K^{-} \pi^{+} \pi^{+} \pi^{-}$[D23]. Two-dimensional likelihood contours are provided in the paper; here we simply quote $R_{K \pi \pi^{0}}=0.84 \pm 0.07, \delta_{K \pi \pi^{0}}=\left(227_{-17}^{+14}\right)^{\circ}$, and $R_{K 3 \pi}=0.33_{-.023}^{+0.20}, \delta_{K 3 \pi}=\left(114_{-23}^{+26}\right)^{\circ}$. The $K \pi \pi^{0}$ state is highly coherent, while the $K 3 \pi$ state is noticeably less coherent. In 2012, results for $K_{S} K^{+} \pi^{-}$followed [R4]: $R_{K_{S} K \pi}=0.73 \pm 0.08, \delta_{K_{S} K \pi}=$ $(8.3 \pm 15.2)^{\circ}$; results were also presented for a restricted $K^{*}$ region. The quantities $c_{i}, s_{i}$ for $K_{S} \pi^{+} \pi^{-}$and related modes, needed for the GGSZ method, have also been presented in 2009 and 2010 [25, [26]. The first paper uses $K_{L} \pi^{+} \pi^{-}$events to improve statistics, while the second analyzes both $K_{S, L} \pi^{+} \pi^{-}$and $K_{S, L} K^{+} K^{-}$. For $K_{S} \pi \pi$, the key results are values of $c_{i}, s_{i}$ in eight bins across the Dalitz plot. All of the results in this paragraph have already been used in measurements of $\gamma$ with $B \rightarrow D K$ decays [ [ 8$]$.

While not a quantum correlation analysis, CLEO-c also investigated the SCS mode $K^{+} K^{-} \pi^{+} \pi^{-}$. In prior studies, resonance structures were investigated, but data was not flavor-tagged: $D^{0}$ and $\bar{D}^{0}$ decays were mixed together. This made estimates of the power of this mode for $\gamma$ analyses uncertain [27]. Isobar model fits to the flavor-separated samples from CLEO-c greatly improved the ability to forecast this mode's prospects for use in extracting $\gamma$ [28]].

We now turn to several more recent results. The first is a "CLEO-c legacy" result; i.e., one performed by a small subset of the former collaboration using the legacy dataset. It is an update to the $K^{-}(n \pi)^{+}$Atwood-Soni coherence factor analysis [ㅁ] ]. The main improvements are inclusion of $K_{S} \pi^{+} \pi^{-}$tags and use of updated external inputs (branching fractions, mixing parameters, $K \pi$ strong phase). The updated results are now $R_{K \pi \pi^{0}}=0.82 \pm 0.07, \delta_{K \pi \pi^{0}}=\left(164_{-14}^{+20}\right)^{\circ}$, and $R_{K 3 \pi}=$ $0.32_{-0.28}^{+0.20}, \delta_{K 3 \pi}=\left(225_{-78}^{+21}\right)^{\circ}$.

Another CLEO-c legacy concerns the $C P$ purity of the $\pi^{+} \pi^{-} \pi^{0}$ and $K^{+} K^{-} \pi^{0}$ final states [B]] . Specifically, the $C P+$ fraction, $F_{+}=N_{+} /\left(N_{+}+N_{-}\right)$, was measured, where $N_{+,-}$are the normalized yields for the $C P+, C P$ - components of the signal decays. These are determined using $C P$ tags and the knowledge the only unlike $C P$ combinations are allowed. They find $F_{+}\left(\pi^{+} \pi^{-} \pi^{0}\right)=$ $0.968 \pm 0.017 \pm 0.006$ and $F_{+}\left(K^{+} K^{-} \pi^{0}\right)=0.731 \pm 0.058 \pm 0.021$. The $F_{+}$value for the $3 \pi$ mode is close enough to 1 that it essentially acts like the 2-body GLW $C P$-eigenstates, with a modest dilution effect. In particular, this dilution will be $D=\left(N_{+}-N_{-}\right) /\left(N_{+}+N_{-}\right)=2 F_{+}-1$. Later, the $\pi^{+} \pi^{-} \pi^{+} \pi^{-}$final state was similarly analyzed, resulting in $F_{+}\left(\pi^{+} \pi^{-} \pi^{+} \pi^{-}\right)=0.737 \pm 0.028$ [B]]. In addition, the previous $h^{+} h^{-} \pi^{0}$ results were redone to include the use of $K_{S, L} \pi^{+} \pi^{-}$tags. Updated results, superseding the previous results, were reported: $F_{+}\left(\pi^{+} \pi^{-} \pi^{0}\right)=0.973 \pm 0.017$ and $F_{+}\left(K^{+} K^{-} \pi^{0}\right)=0.732 \pm 0.055$.

As noted in the introduction, mixing is also a possible source of useful information. Indeed, CLEO legacy data has now been combined with LHCb data, improving results on $R_{K n \pi}$ [B2]. The

\footnotetext{
${ }^{2} \mathrm{The} K_{S} K \pi$ analysis adds in $15 \mathrm{fb}^{-1}$ of continuum charm data taken near $10 \mathrm{GeV}$, while the $K K \pi \pi$ results use 24 $\mathrm{fb}^{-1}$ of such continuum charm and $0.6 \mathrm{fb}^{-1}$ of data taken at $4170 \mathrm{MeV}$.
} 
authors find $R_{K \pi \pi^{0}}=0.81_{-0.06}^{+0.07}$ and $R_{K 3 \pi}=0.32_{-0.08}^{+0.12}$. The LHCb mixing data are for the $k K 3 \pi$ state, and hence that $R$ value benefits from a reduction in error of about 2.5 times, while the other mode is almost unaffected. Many interesting contour plots from the multi-parameter fit are included in the paper.

BESIII has now entered the game with their larger dataset, recently publishing a $K^{-} \pi^{+}$strongphase result [B3]. The analysis is simpler than the related CLEO-c result, and concentrates on the effect of the relative $K \pi$ strong phase on the $C P$-tagged branching ratio asymmetry

$$
A_{K \pi}^{C P}=\frac{\mathscr{B}\left(D_{C P-} \rightarrow K \pi\right)-\mathscr{B}\left(D_{C P+} \rightarrow K \pi\right)}{\mathscr{B}\left(D_{C P-} \rightarrow K \pi\right)+\mathscr{B}\left(D_{C P+} \rightarrow K \pi\right)}
$$

The directly observed asymmetry is $A_{K \pi}^{C P}=(12.7 \pm 1.3 \pm 0.7) \%$. With external inputs for mixing parameters, they then extract $\cos \delta_{K \pi}=1.02 \pm 0.11 \pm 0.06 \pm 0,01$ where the last error is from those inputs. The statistical error dominates and is about $60 \%$ of the final CLEO-c result.

Finally, there is a preliminary BESIII result on the GGSZ $c_{i}, s_{i}$ parameters for $K_{S} \pi^{+} \pi^{-}$[34]. The statistical errors are improved relative to CLEO-c due to the factor of over 3.5 increase in the available integrated luminosity. As with CLEO-c, $K_{L} \pi^{+} \pi^{-}$events are used to improve statistics. Final results for use in $\gamma$ extraction should be available soon.

\section{Selected Issues}

As extractions of $\gamma$ with these methods become more accurate, care must be taken to use accurate calculations of relevant rates. We discuss a selection of relevant issues next.

With larger threshold datasets, one needs to carefully consider if inclusion of the $K_{L} h^{+} h^{-}$ modes in the $K_{S} h^{+} h^{-}$analyses brings in unwanted model-dependence.

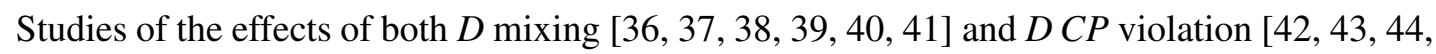
45] seem to be essentially complete now. In general, one can include effects where relevant in the $\gamma$ analyses. I note that issues of $C P$ violation and mixing in the $B$ system have also been explored, but these are beyond the scope of this review.

In modes that involve $K_{S}$, one must also take care with kaon $C P$ violation and mixing. Such effects have been topical recently in the context of $\tau$ and $D$ physics [46, 47]; in particular, sensitivity to the proper-time acceptance of the $K_{S} \rightarrow \pi^{+} \pi^{-}$decays was highlighted. More recently, the issues specific to $\gamma$ extractions have also been treated [48]].

A further complication arises due to interactions of kaons with material. Different $K^{0}, \bar{K}^{0}$ interaction rates lead to coherent regeneration, as explored in Ref. [49] in the context $C P$ violation searches in $B, D$ decays. Further attention is needed here for $\gamma$ : effects are experiment-specific not only because of acceptance, as with the previous issue, but now also due to material details as well.

Another concern involves the required efficiency corrections across the $D$ Dalitz plots. They are used when analyzing charm data in order to quote idealized quantities $\left(R, \delta\right.$, or $\left.c_{i}, s_{i}\right)$ with efficiency effects removed. Similarly, different efficiency effects need to be accounted for when applying these quantities to the $B$ factory data. The issue is the accuracy of such corrections and associated systematic uncertainties as overall precision continues to improve. This likely deserves further attention. 
Phases are also relevant for $D$ mixing; analyses aim to measure the normalized mass and lifetime differences between the physical eigenstates: $x=\Delta m / \bar{\Gamma}$ and $y=\Delta \Gamma / 2 \bar{\Gamma}$, where $\bar{\Gamma}=$ $\left(\Gamma_{1}+\Gamma_{2}\right) / 2$. But the most powerful analyses use hadronic final states with both CF and DCSD amplitudes. In the simplest case, $D \rightarrow K \pi$, the measured parameters are $x, y$ rotated by the relative strong phase. In the case of multi-body decays, there is a similar rotation; see, for example, the BaBar result for $D$ mixing extracted via $K^{-} \pi^{+} \pi^{0}$ [50]. Here, the rotation is a more complicated Dalitz-averaged effect, analogous to effects we have discussed for $\gamma$ analyses. Currently, threshold data have not yet been analyzed in a way that allows one to easily un-rotate such multi-body results. However, model-independent methods are discussed in Ref. [40].

More attention should also be paid to of the potential to extract coherence factors from $D$ mixing analyses [ $[\mathbf{]}]$, based on samples taken along with $B$ physics data by both $e^{+} e^{-}$and hadron colliders. Such results can complement, or even surpass, charm threshold data. We noted one such example earlier (which combined threshold data and mixing [32]); more work along these lines is expected.

\section{Conclusions}

The physics of the quantum coherence of charm at threshold is both intellectually interesting on its own and also of great practical importance to weak interaction physics and CKM matrix studies. In particular, its role as a source of inputs to extractions of the CKM angle $\gamma$ from $B$ physics remains a very topical and active area. New ideas, such as leveraging charm mixing as an alternate source of such inputs help to keep the field exciting. And while many issues relevant to accuracy and controlling systematic uncertainties have been addressed ( $D$ mixing and CPV), some other issues (efficiency across Dalitz plots and $K$ regeneration) appear to me at least to be less fully explored. Perhaps this review will inspire more work and new workers to help us move forward.

\section{Acknowledgments}

The author would like to thank his colleagues on CLEO-c and BESIII for their hard work on results reported here and also his new colleagues on BelleII for their interest in this physics. Special thanks to Onur Albayrak, Alex Bondar, Tim Gershon, Jim Libby, Alexey Petrov, Guy Wilkinson and Jure Zupan for helpful conversations, and to Angelo Carbone and his team for a quite successful and enjoyable workshop. Most of this review appeared previously as electronic proceedings of the CKM2014 meeting in Vienna [52]; as such, thanks are also due to Christoph Schwanda and company for organizing that very beneficial meeting.

\section{References}

[1] M. Goldhaber and J. L. Rosner, Phys. Rev. D 15, 1254 (1977).

[2] Z.-Z. Xing, Phys. Rev. D 55, 196 (1997).

[3] M. Gronau, Y. Grossman and J. L. Rosner, Phys. Lett. B 508, 37 (2001).

[4] D. Atwood and A. A. Petrov, Phys. Rev. D 71, 054032 (2005) [hep-ph/ 0207165$].$ 
[5] D. M. Asner and W. M. Sun, Phys. Rev. D 73, 034024 (2006); E: ibid, D 77, 019901 (2008).

[6] I. I. Bigi and A. I. Sanda, Phys. Lett. B 211, 213 (1988).

[7] J. Brod and J. Zupan, JHEP 01, 051 (2014).

[8] See, for example, the summary of measurements maintained by the Heavy Flavor Averaging Group at http://www.slac.stanford.edu/xorg/hfag/triangle/ .

[9] D. Atwood and A. Soni, Phys. Rev. D 68, 033003 (2003).

[10] M. Gronau and D. London, Phys. Lett. B 253, 483 (1991).

[11] M. Gronau and D. Wyler, Phys. Lett. B 265, 172 (1991).

[12] D. Atwood, G. Eilam, M. Gronau, and A. Soni, Phys. Lett. B 341, 372 (1988).

[13] D. Atwood, I. Dunietz and A. Soni, Phys. Rev. Lett. 78, 3257 (1997).

[14] D. Atwood, I. Dunietz and A. Soni, Phys. Rev. D 63, 036005 (2001).

[15] A. Bondar, Proceedings of BINP Special Analysis Meeting on Dalitz Analysis, Novosibirsk, 24-26 Sep. 2002 (unpublished).

[16] A. Giri, Y. Grossman, A. Soffer and J. Zupan, Phys. Rev. D 68, 054018 (2003).

[17] A. Bondar and A. Poluektov, Eur. Phys. J. C 47, 347 (2006).

[18] A. Bondar and A. Poluektov, Eur. Phys. J. C 55, 51 (2008).

[19] Y. Grossman, Z. Ligeti and A. Soffer, Phys. Rev. D 67, 071301(R) (2003).

[20] A. Bondar and T. Gershon, Phys. Rev. D 70, 091503(R) (2004).

[21] CLEO Collaboration, J. L. Rosner et al., Phys. Rev. Lett. 100, 221801 (2008), D. M. Asner et al., Phys. Rev. D 78, 012001 (2008).

[22] CLEO Collaboration, D. M. Asner et al., Phys. Rev. D 86, 112001 (2012).

[23] CLEO Collaboration, N. Lowrey et al., Phys. Rev. D 80, 031105 (2009).

[24] CLEO Collaboration, J. Insler et al., Phys. Rev. D 85, 092016 (2012).

[25] CLEO Collaboration, R. A. Briere et al., Phys. Rev. D 80, 032002 (2009).

[26] CLEO Collaboration, J. Libby et al., Phys. Rev. D 82, 112006 (2010).

[27] J. Rademacker and G. Wilkinson, Phys. Lett. B 647, 400 (2007).

[28] CLEO Collaboration, M. Artuso et al., Phys. Rev. D 85, 122002 (2012).

[29] J. Libby et al., Phys. Lett. B 731, 197 (2014).

[30] M. Nayak et al., Phys. Lett. B 740, 1 (2015).

[31] S. Malde et al., Phys. Lett. B 747, 9 (2015).

[32] T. Evans et al., Phys. Lett. B 757, 520 (2016).

[33] BESIII Collaboration, M. Ablikim et al., Phys. Lett. B 734, 227 (2014).

[34] BESIII Collaboration, preliminary results presented by D. Ambrose, APS Meeting, April 2014.

[35] I. I. Bigi and H. Yamamoto Phys. Lett. B 349, 363 (1995).

[36] C. C. Meca and J. P. Silva, Phys. Rev. Lett. 81, 1377 (1998). 
[37] A. Amorim, M. G. Santos and J. P. Silva, Phys. Rev. D 59, 056001 (1999).

[38] J. P. Silva and A. Soffer, Phys. Rev. D 61, 112001 (2000).

[39] Y. Grossman, A. Soffer and J. Zupan, Phys. Rev. D 72, 031501(R) (2005).

[40] A. Bondar, A. Poluektov and V. Vorobiev, Phys. Rev. D 82, 034033 (2010).

[41] M. Rama, Phys. Rev. D 89, 014021 (2014).

[42] W. Wang, Phys. Rev. Lett. 110, 061802 (2013).

[43] M. Martone and J. Zupan, Phys. Rev. D 87, 034005 (2013).

[44] B. Bhattacharya, M. Gronau, D. London and J. L. Rosner, Phys. Rev. D 87, 074002 (2013)

[45] A. Bondar, A. Dolgov, A. Poluektov and V. Vorobiev, Eur. Phys. J. C 73, 2476 (2013).

[46] I. I. Bigi and A. I. Sanda, Phys. Lett. B 625, 47 (2005).

[47] Y. Grossman and Y. Nir, JHEP 04, 002 (2012).

[48] Y. Grossman and M. Savastio, JHEP 03, 008 (2014).

[49] B. R. Ko, E. Won, B. Golob and P. Pakhlov, Phys. Rev. D 84, 111501(R) (2011).

[50] BaBar Collaboration, B. Aubert et al., Phys. Rev. Lett. 103, 211801 (2009).

[51] S. Harnew and J. Rademacher, Phys. Lett. B 728, 296 (2014).

[52] R. A. Briere, Quantum Correlated Charm at Threshold and Inputs to Extractions of $\gamma$ from B Decays, Proceedings of the 8th International Workshop on the CKM Unitarity Triangle (CKM 2014), Vienna, Austria, 2014 [arXiv: 1411 . 7327]. 